EPJ manuscript No.

(will be inserted by the editor)

\title{
Hadrons in Hot and Dense Matter
}

\author{
Ralf Rapp ${ }^{1}$ a \\ Dept. of Physics and Astronomy, SUNY Stony Brook, New York 11794-3800, U.S.A. \\ Received: date / Revised version: date
}

\begin{abstract}
The description of excitations in hot and dense (hadronic) matter is discussed with emphasis on the use of correlation functions as a common framework for comparing different model (and QCD lattice) calculations with each other. Typical regimes of applicability of hadronic approaches are assessed, together with possibilities to confront them with experiment. We also elaborate on recent developments to relate baryonic in-medium effects to chiral symmetry restoration.
\end{abstract}

PACS. 25.75.-q Relativistic Heavy-Ion Collisions - 21.65.+f Nuclear Matter

\section{Introduction}

The study of the phase structure of strong interactions (i.e., QCD phase diagram) not only consists of determining the correct ground state, but also of identifying the relevant excitations, which are, after all, the probes amenable to experiment. At low to intermediate matter densities, this corresponds to the widely investigated topic of in-medium modifications of the hadronic spectrum as we know it from the vacuum. When passing through a

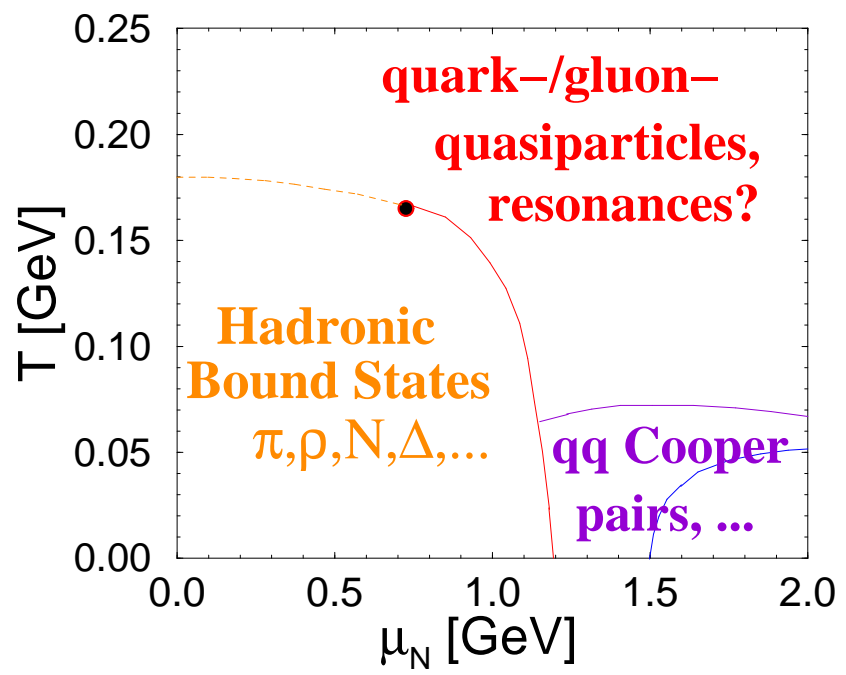

Fig. 1. Schematic phase structure and pertinent excitations of the QCD phase diagram.

\footnotetext{
a Present address: NORDITA, Blegdamsvej 17, DK-2100 Copenhagen; work supported in part by US DOE grant no. DE-FG02-88ER40388.
}

phase transition (or cross-over region) an important question then is how changes in the ground state symmetries (or order parameters) reflect themselves in the spectrum, schematically sketched in Fig. 11. A general approach to the problem obviously requires a common framework which allows to compare results of individual models.

\section{Correlation and Spectral Functions}

\subsection{Definition}

The general description of an excitation in a given quantum-number channel $\alpha$ can be formulated by correlation functions of the pertinent current operators [1], averaged over the thermodynamic partition function at temperature $T$ and (baryon-) chemical potential $\mu_{B}$. In coordinate space one has

$$
\begin{aligned}
\Pi_{\alpha}(x) & =\operatorname{tr}\left(\left[j_{\alpha}(x) j_{\alpha}^{\dagger}(0)\right] \mathrm{e}^{-\left(\hat{H}-\mu_{B} \hat{N}\right) / T}\right) / \mathcal{Z} \\
& =\int \frac{d^{4} q}{(2 \pi)^{4}} \Pi_{\alpha}(q) \mathrm{e}^{-i q x} .
\end{aligned}
$$

Its interpretation becomes more transparent in momentum space: in the timelike region, $M^{2}=q_{0}^{2}-\boldsymbol{q}^{2}>0$, the "spectral function" $\operatorname{Im} \Pi_{\alpha}$ contains the information on the physical excitation spectrum, whereas the spacelike region, $M^{2}<0$, encodes screening phenomena as well as fluctuation and susceptibility properties (see Refs. 2, 3. for recent examples in scalar and vector channel).

In lattice QCD one typically evaluates $\Pi_{\alpha}(x)$ in (Euclidean) coordinate space; at finite temperatures, the transformation into Minkowski space is complicated by the shrinking of the "Matsubara box" $\tau \epsilon\{0,1 / T\}$ (due to periodic boundary conditions). However, model calculations in Minkowski space can, in principle, be straightforwardly transformed into Euclidean space for direct comparison with lattice data, see Sect. 3.2 below. 
Ralf Rapp: Hadrons in Hot and Dense Matter

\subsection{Vacuum: Chiral Breaking vs. Confinement}

Correlation functions in free space are determined by the propagation and interaction of anti-/quarks in the nonperturbative QCD vacuum, which is characterized by two main phenomena, confinement and spontaneous breaking of chiral symmetry (SBCS). Whereas an understanding of the former is still elusive, the latter is widely believed to be due to instantons, which successfully reproduce QCD vacuum properties as well as low-lying meson and baryon correlation functions, see, e.g., Ref. [4. However, attempts to obtain confinement from instantons have failed so far. On the other hand, the well-known Reggebehavior of hadronic masses, $J \propto M^{2}$, is suggestive for string-like excitations associated with a confining force. Furthermore, at masses $M \geq 2 \mathrm{GeV}$, hadronic chiral partners seem to develop a degeneracy pattern suggestive for an "effective" restoration of chiral symmetry 5 . Most of these features are indeed supported by the appearance of the baryonic spectrum as extracted from the particle data group [6], cf. Fig. 2.

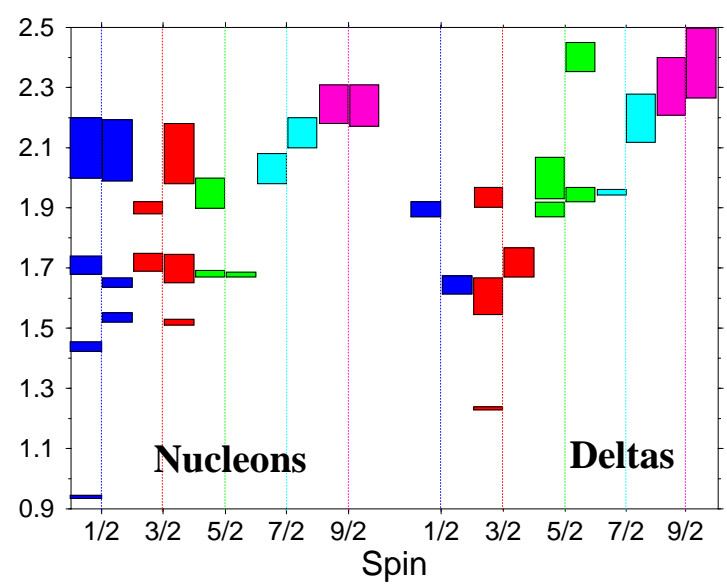

Fig. 2. Baryon spectrum according to PDG [6]. For given spin, left and right column correspond to positive and negative parity, respectively. Note the mass gaps of $\Delta E \simeq 0.5 \mathrm{GeV}$ for low-lying spin- $1 / 2$ and $-3 / 2$ states, and the approximate degeneracy at higher masses, especially for $J \geq 5 / 2$.

\subsection{In Medium: Hadronic Approaches}

Various methods to address medium effects on hadronic properties (and the equation of state) are available, cf. Table 1 .

At low densities and/or temperatures, systematic expansions using chiral perturbation theory [7] or a reduction formalism [B] can be performed.

At intermediate hadronic densities, as, e.g., encountered in the later stages of heavy-ion collisions, many-body effects necessitating (partial) resummations, become important. E.g., in the vector $\left(J^{P}=1^{-}\right)$channel, the "melting" of the $\rho$-meson [9] enables to describe the dilepton excess observed at CERN-SPS energies [10] (cf. Ref. [11]
Table 1. Applicability domains of approaches to hot+dense matter (ex = extrapolation, $n_{0}=0.16 \mathrm{fm}^{-3}$ ).

\begin{tabular}{l|llll}
\hline$T[\mathrm{MeV}]$ & $\mid \leq 120$ & $120-150$ & $150-T_{c}$ & $\geq T_{c}$ \\
\hline$\varepsilon\left[\mathrm{GeV} / \mathrm{fm}^{3}\right]$ & $\mid \leq 0.05$ & $0.05-0.3$ & $0.3-0.8$ & $\geq 1$ \\
\hline$n_{\text {had }}\left[n_{0}\right]$ & $\mid \leq 0.5$ & $0.5-2$ & $2-5$ & N $/ \mathrm{A}$ \\
\hline Method & $\chi$ PT & many-body & had-ex & pQCD-ex \\
\hline
\end{tabular}

for an alternate view, and Ref. [12] for a review). Likewise, in the scalar $\left(0^{+}\right)$channel, nuclear many-body effects contribute significantly to the threshold enhancement found in two-pion production experiments on nuclei 13.

In the expected phase transition region, both hadronic (below $T_{c}$ ) and partonic (above $T_{c}$ ) approaches have to rely on extrapolations. An important realization is, however, that medium modifications of light hadrons already encode precursor phenomena of chiral symmetry restoration. This connection can be made explicit by addressing the modifications of the respective chiral partners on an equal footing (e.g., $\left.\pi-\sigma, \rho-a_{1}, N-N^{*}(1535)\right)$.

\section{Recent Applications: Axial-/Vector Channel}

\subsection{Dileptons and $a_{1}$ Spectral Function}

There is general consensus that the large excess in lowmass $(M \leq 1 \mathrm{GeV})$ dilepton production observed in central $P b(158 \mathrm{AGeV})+P b$ collisions by CERES/NA45 10] is due to in-medium radiation from strongly modified $\rho$-mesons. The importance of baryon-induced effects [9] is supported by an even larger (relative) enhancement at lower SPS energies (40 AGeV), cf. Fig. 3. The final interpretation on the nature of chiral restoration is still under debate. As argued above, this requires the evaluation of the in-medium axialvector spectral function. Such an analysis has been

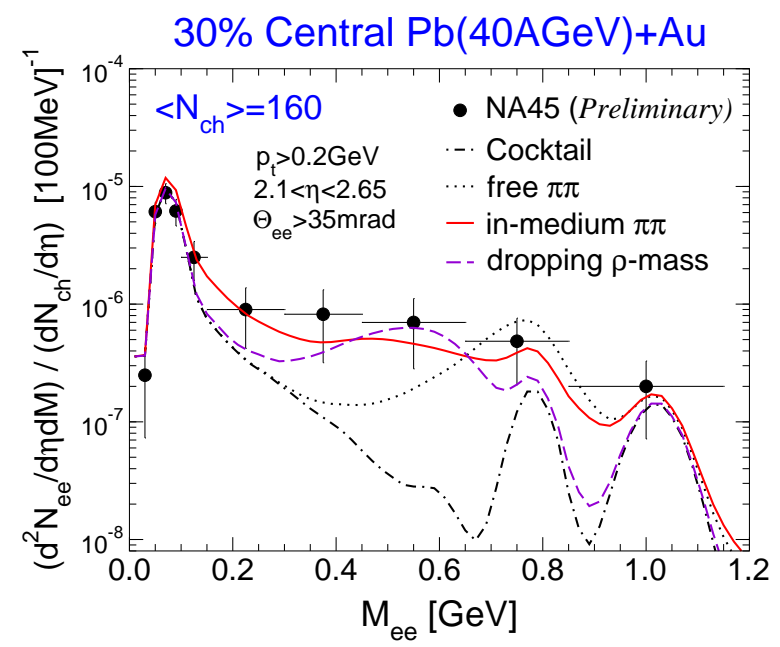

Fig. 3. Theoretical predictions using in-medium $\rho$ mesons 9 compared to recent NA45 data [10]. 


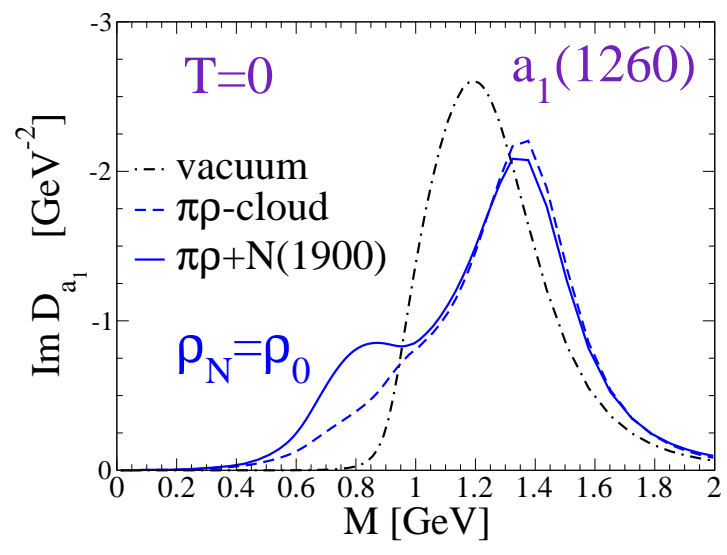

Fig. 4. $a_{1}$ spectral function in vacuum (dotted line) and at normal nuclear matter density.

performed, e.g., in Ref. [14 for a thermal $\pi \sigma \rho a_{1}$ gas. However, even in a net baryon-free environment, the presence of baryon-antibaryon pairs at temperatures close to $T_{c}$ has appreciable impact on the $\rho$ spectral function 15. Preliminary results for the $a_{1}(1260)$ within a many-body framework at finite density are shown in Fig. 4. In addition to an in-medium modified $\pi \rho$-cloud, significant contributions arise from $a_{1} \rightarrow N^{*}(1900) N^{-1}$ excitations, the putative $S U(2)$ chiral partner of the $\rho \rightarrow N(1520) N^{-1}$ bubble. Experimental information on the decay modes of baryon resonances, here $N(1900) \rightarrow N a_{1}$ (possibly measurable with the HADES detector using $\pi$-beams at GSI), would be of great help to constrain such calculations.

\subsection{Vector Correlator in Euclidean Space}

We finally return to the question of model comparisons with lattice-QCD results. In a "mixed" representation, the temporal Euclidean correlator is related to the spectral function in Minkowski space via

$$
\Pi_{\alpha}(\tau, q)=\int_{0}^{\infty} \frac{d q_{0}}{\pi} \operatorname{Im} \Pi_{\alpha}\left(q_{0}, q\right) \frac{\cosh \left(q_{0}\left[\tau-\frac{1}{2 T}\right]\right)}{\sinh \left(q_{0} / 2 T\right)}
$$

A quenched lattice analysis of the vector correlator at zero 3-momentum, $\Pi_{V}(\tau, q=0)$, above $T_{c}$ has recently been presented in Ref. 16. For illustration, we show in Fig. 5 results for the same quantity using the sum of (free and inmedium) $\rho$ and $\omega$ spectral functions, supplemented with a perturbative continuum above $q_{0} \simeq 1.3 \mathrm{GeV}$. We stress that the enhancement at large $\tau T \simeq 0.5$ is at the origin of the dilepton excess at low-mass (cf. solid line in Fig. 3 ).

\section{4 (Some) Open Issues}

The investigation of hadronic excitations and their medium modifications provides important insights into nonperturbative properties of the QCD phase diagram. In vacuum, confinement and chiral symmetry breaking establish

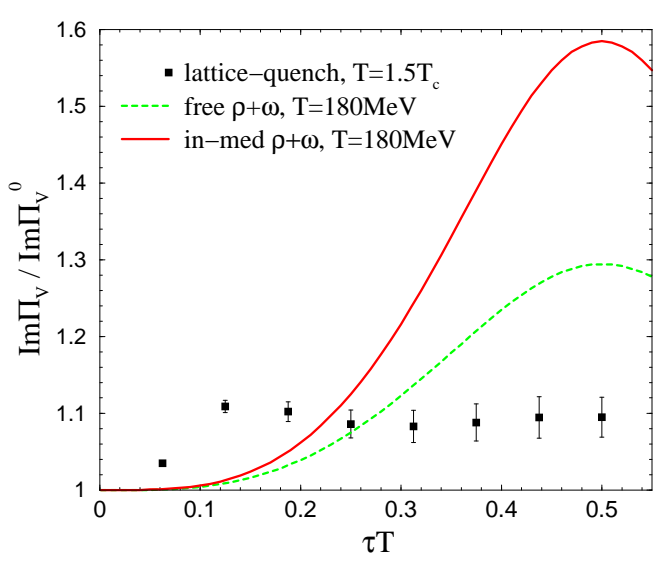

Fig. 5. Temporal vector correlator at $T=180 \mathrm{MeV}$ (normalized to the $\mathcal{O}\left(\alpha_{s}^{0}\right)$ pQCD result) obtained from a hadronic model for $\rho$ and $\omega$ spectral functions 15] in vacuum (dotted line) and with medium effects at $\mu_{B} \simeq 25 \mathrm{MeV}$ (solid line). Quenched lattice results (squares) at $T=1.5 T_{c}$ are from Ref. [16].

themselves in different (mass-) regimes of the spectrum, whereas associated phase transitions seem to occur at the same critical temperature (whether this holds true at high baryon densities is an open question). In particular, the role of (baryonic) resonances requires further understanding: on the one hand, they importantly figure into medium effects on, e.g., light (axial-) vector mesons (as inferred from dilepton spectra) and thus into (the approach towards) chiral restoration; on the other hand, their copious population close to $T_{c}$ significantly reduces the "jump" in the number of degrees of freedom in the transition to an (almost) ideal Quark-Gluon Plasma. Hadronic model calculations can thus be expected to remain an important tool to interpret both experimental and lattice data.

\section{References}

1. E.V. Shuryak, Rev. Mod. Phys. 65, 1 (1993).

2. G. Chanfray and M. Ericson, hucl-th/0106069.

3. M. Prakash et al., Phys. Rev. C65, 034906 (2002).

4. T. Schäfer and E. Shuryak, Phys. Rev. D54, 1099 (1996).

5. T. Cohen and L. Glozman, Phys. Rev. D65, 016006 (2001).

6. Particle Data Group, Eur. Phys. J. C15, 1 (2000).

7. N. Kaiser, S. Fritsch and W. Weise, Nucl. Phys. A697, 255 (2002).

8. J. Steele, H. Yamagishi and I. Zahed, Phys. Rev. D56, 5605 (1997).

9. R. Rapp and J. Wambach, Eur. Phys. J. A6, 415 (1999).

10. CERES Collaboration (D. Adamova et al.), Nucl. Phys.

A698, 253 (2002); J. Wessels, these proceedings.

11. G.E. Brown and M. Rho, nucl-th/0206021.

12. R. Rapp and J. Wambach, Adv. Nucl. Phys. 25, 1 (2000).

13. M.J. Vicente-Vacas and E. Oset, hucl-th/0204055, and these proceedings.

14. M. Urban, M. Buballa and J. Wambach, Phys. Rev. Lett. 88, 042002 (2002).

15. R. Rapp, Phys. Rev. C63, 054907 (2001).

16. F. Karsch et al., Phys. Lett. B530, 147 (2002). 